\title{
Diacronie
}

Studi di Storia Contemporanea

$\mathrm{N}^{\circ} 28,4 \mid 2016$

La voce del silenzio

Prigionieri russi, prigionieri in Russia: detenzione, tradimento e spionaggio nella percezione e nelle strategie dei servizi segreti austro-ungarici (1914-1918)

\section{Verena Moritz}

Traduttore: Alessandro Salvador

\section{(2) OpenEdition}

\section{Journals}

\section{Edizione digitale}

URL: http://journals.openedition.org/diacronie/4677

DOI: 10.4000/diacronie.4677

ISSN: 2038-0925

\section{Editore}

Association culturelle Diacronie

\section{Notizia bibliografica digitale}

Verena Moritz, « Prigionieri russi, prigionieri in Russia: detenzione, tradimento e spionaggio nella percezione e nelle strategie dei servizi segreti austro-ungarici (1914-1918) », Diacronie [Online], N2 28, 4 | 2016, documento 1, Messo online il 29 décembre 2016, consultato il 01 mai 2019. URL : http:// journals.openedition.org/diacronie/4677; DOI : 10.4000/diacronie.4677 


\section{Diacronie}

N. 28 | 4|2016 La voce del silenzio: intelligence, spionaggio e conflitto nel XX secolo

1/

\section{Prigionieri russi, prigionieri in Russia: detenzione, tradimento e spionaggio nella percezione e nelle strategie dei servizi segreti austroungarici (1914-1918)}

Verena MORITZ*

Traduzione di Alessandro SALVADOR

Durante la prima guerra mondiale centinaia di migliaia di soldati dell'esercito austroungarico caddero in mani nemiche. Allo stesso modo, un gran numero di soldati nemici fu catturato dalle forze armate della monarchia danubiana. I servizi segreti militari austroungarici gestirono questa situazione in modi diversi: i soldati catturati erano visti sia come "rischio per la sicurezza", sia come "fonte d'informazione". Usando l'esempio degli austroungarici tornati dalla prigionia in Russia e dei prigionieri di guerra russi detenuti dalla doppia monarchia, questo articolo mira a mostrare come $i$ servizi segreti dell'Austria-Ungheria affrontarono la sfida di distinguere tra "fiducia e sfiducia". Questo articolo si sofferma inoltre nel valutare se $i$ servizi segreti di un impero multinazionale dovessero affrontare problemi particolari nell'ottenere informazioni o prevenire lo spionaggio.

\section{Premessa}

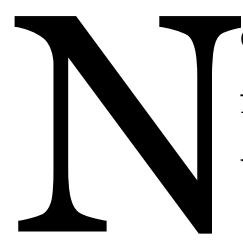

el corso della Prima guerra mondiale, centinaia di migliaia di soldati finirono prigionieri dell'esercito austro-ungarico. Allo stesso modo, anche un numero consistente di militari austroungarici finirono nelle mani del

\footnotetext{
${ }^{1}$ Il presente articolo si basa sulle ricerche compiute nel febbraio del 2016 nell'ambito di un progetto finanziato dal Fonds zur Förderung der wissenschaftlichen Forschung (FWF) austriaco.
} 
nemico ${ }^{2}$. Tra 1,86 e 2,3 milioni di soldati nemici si trovavano sotto la custodia dell'impero asburgico, mentre le truppe zariste imprigionarono circa 2,4 milioni di combattenti degli eserciti avversari3 ${ }^{3}$. Tra i prigionieri custoditi in Austria-Ungheria, i russi costituivano la stragrande maggioranza (tra 1.269.000 e 1.750.000) 4 e in Russia, allo stesso modo, erano i soldati austroungarici a costituire la massa dei prigionieri (circa 2.100.000)5. La logistica e l'approvvigionamento dei detenuti crearono gravi problemi ad entrambi gli imperi multinazionali ${ }^{6} \mathrm{e}$, inoltre, la presenza di soldati nemici nel proprio territorio o nelle aree controllate dal proprio esercito era collegata a numerose questioni di sicurezza, tra le quali lo spionaggio era una delle più importanti. Informazioni sulla consistenza delle truppe, il numero e la qualità degli equipaggiamenti e sui piani operativi non dovevano, in nessun caso, finire nelle mani del nemico7.

Quanto era, però, preparata la popolazione civile, che dovette confrontarsi fin dall'inizio della guerra con un numero crescente di soldati nemici, ad affrontare pericoli come la diffusione involontaria di informazioni? Tematiche come quella dello spionaggio e del tradimento erano presenti sulla stampa austroungarica già da lungo tempo prima dello scoppio del conflitto in connessione con la presenza contestuale del "nemico" nella veste di prigioniero di guerra. Negli anni precedenti alla Prima guerra mondiale, la popolazione della doppia monarchia dovette confrontarsi con numerosi casi di spionaggio ed assistere ad entusiasmanti processi per spionaggio che, in buona parte, riguardavano propri concittadini. Sono da menzionare, tra gli altri, il processo

\footnotetext{
${ }^{2}$ Sul problema delle cifre dei prigionieri di guerra nella Prima guerra mondiale si veda: NACHTIGAL, Reinhard, «Zur Anzahl der Kriegsgefangenen im Ersten Weltkrieg», in Militärgeschichtliche Zeitschrift : Der Erster Weltkrieg, 67, 2/2008. pp. 345-384., p. 359.

${ }^{3}$ NACHTIGAL, Reinhard, RADAUER, Lena, Prisoners of War (Russian Empire), in DANIEL, Ute, GATRELL, Peter, JANZ, Oliver, JONES, Heather, KEENE, Jennifer, KRAMER, Alan, NASSON, Bill (ed. by), 1914-1918-online. International Encyclopedia of the First World War, Berlin, Freie Universität Berlin, 2014, URL: < http://encyclopedia.1914-1918online.net/article/prisoners_of_war_russian_empire > [consultato il 28 dicembre 2016].

${ }^{4}$ MORITZ, Verena, WALLECZEK-FRITZ, Julia, Prisoners of War (Austria-Hungary), in DANIEL, Ute, GATRELL, Peter, JANZ, Oliver, JONES, Heather, KEENE, Jennifer, KRAMER, Alan, NASSON, Bill (ed. by), 1914-1918-online. International Encyclopedia of the First World War, Berlin, Freie Universität Berlin, 2014, URL: < http://encyclopedia.1914-1918online.net/article/prisoners_of_war_russian_empire > [consultato il 28 dicembre 2016]. Vedi anche: MORITZ, Verena, Zwischen Nutzen und Bedrohung. Die russischen Kriegsgefangenen in Österreich 1914-1921, Bonn, Bernard \& Graefe Verlag, 2005, pp. 329-330.

${ }^{5}$ WURZER, Georg, Die Kriegsgefangenen der Mittelmächte in Rußland im Ersten Weltkrieg, Tübingen, V\&R unipress, 2000, p. 22.

${ }^{6}$ MORITZ, Verena, Zwischen Nutzen und Bedrohung, cit., pp. 66-89.

${ }^{7}$ Sulle questioni di sicurezza relative al trasporto di prigionieri in Austria-Ungheria si veda: MORITZ, Verena, Kriegsgefangene als "Sicherheitsproblem“ - Zur Kontrolle von „Feindsoldaten“ im urbanen Raum (1914-1920), in GRUBER, Elisabeth, WEIGL, Andreas (Hg.), Stadt und Gewalt, Innsbruck-Wien-Bozen, Studienverlag, 2016, pp. 269-286.
} 
contro i cosiddetti "russofili" a Marmaros-Sziget e a Lemberg8 . Nella stampa degli anni prebellici si trovavano articoli regolari e completi sui cosiddetti «casi di tradimento»9. Lo spionaggio si sviluppò come tematica costante a partire dagli anni Novanta dell'Ottocento, con il cosiddetto "caso Dreyfus" e iniziò a coinvolgere non solo la stampa, ma anche la letteratura e il nascente cinema ${ }^{10}$.

Negli anni precedenti alla Prima guerra mondiale l'ufficio dell'intelligence dello stato maggiore dell'esercito evidenziò un costante aumento dei casi di spionaggio. Una situazione tale per cui tutti i possibili scenari di minaccia potessero divenire un pericolo

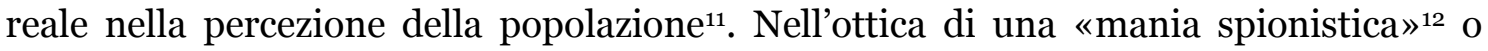
«spionite» ${ }^{13}$ che era già ampiamente diffusa prima dello scoppio della guerra, che fiorì soprattutto in Gran Bretagna ${ }^{14}$, ma che non si limitò neanche nell'impero austroungarico, non sorprende che settori della popolazione civile nei confini multietnici dell'impero finissero sotto sospetto costante di essere spie del nemico. Ufficiali dei servizi segreti avevano anticipato già prima del 1914 che la slealtà dei membri delle diverse nazionalità dell'impero asburgico poteva costituire un grosso rischio per la sicurezza in caso di scoppio di una guerra. Maximilian Ronge, l'ultimo capo dei servizi segreti dell'imperial-regio esercito, espose dopo la guerra l'idea che queste paure all'alba del conflitto erano in buona parte corrette ${ }^{15}$. La corrispondente «pulizia degli spazi di avanzata da elementi politicamente inaffidabili» fu tuttavia vista anche da ufficiali più moderati come assolutamente necessaria. Mentre, però, Ronge riteneva che le dure misure nei confronti, ad esempio, dei "russofili" in Galizia fossero

\footnotetext{
${ }^{8}$ Esaustivo sul tema: BACHMANN, Klaus, „Ein Herd der Feindschaft gegen Rußland“ Galizien als Krisenherd in den Beziehungen der Donaumonarchie mit Rußland (1907-1914), WienMünchen, Oldenbourg, 2001, pp. 241-258.

${ }^{9}$ L'autrice è al momento impegnata nell'analisi di ritagli di giornale su tema dello spionaggio raccolti negli Archivi di Stato austriaci. Sul caso più grave di spionaggio nella monarchia austroungarica prima della guerra si veda: MORITZ, Verena, LEIDINGER, Hannes, Oberst Redl. Der Spionagefall - Der Skandal - Die Fakten, St. Pölten-Salzburg-Wien, Residenz, 2012.

${ }^{10}$ Cfr.: ALTENHÖNER, Florian, 'Spionitis'- reale Korrelate und Deutungsmuster der Angst vor Spionen, 1900-1914, in RAMMERT, Werner, KNAUTHE, Gunther, BUCHENAU, Klaus, ALTENHÖNER, Florian (Hg.), Kollektive Identitäten und kulturelle Innovationen. Ethnologische, soziologische und historische Studien, Leipzig, Universitätsverlag, 2001, pp. 7791. L'autrice sta lavorando ad un articolo sul tema dello spionaggio legato al cinema.

${ }^{11}$ Cfr. MORITZ, Verena, LEIDINGER, Hannes, Im Zentrum der Macht. Die vielen Gesichter des Geheimdienstchefs Maximilian Ronge, St. Pölten-Salzburg, Residenz Verlag, 2007, pp. 65-125.

${ }^{12}$ Cfr. su questo, tra gli altri: FRENCH, David, «Spy fever in Britain 1900-1915», in Historical Journal, 21, 2/1978, pp. 355-370.

${ }^{13}$ Cfr. anche: URBANSKI von Ostrymiecz, August, Spionitis, in LETTOW-VORBECK, Paul (Hg.), Die Weltkriegsspionage, München, Verlag Justin Moser, 1931, pp. 332-338.

${ }^{14}$ Si prenda ad esempio: FRENCH, David, «Spy fever in Britain, 1900-1915», in The Historical Journal, 21, 2/1978, pp. 355-370;ROSE, Andreas, Zwischen Empire und Kontinent. Britische Außenpolitik vor dem Ersten Weltkrieg, München, Oldenbourg, 2011, pp. 82-102.

15 RONGE, Max, Kriegs- und Industriespionage. Zwölf Jahre Kundschaftsdienst, ZürichLeipzig-Wien, Amalthea, 1930, p. 97.
} 
assolutamente corrette, altri le definirono eccessive e controproducenti. August Urbanski, che fino a pochi mesi prima dello scoppio della guerra nel 1914 dirigeva lo stato maggiore dell'ufficio informazioni dell'imperial-regio esercito, associò l'onnipresente paura delle spie con un crescente nervosismo delle truppe combattenti che si rivelò negativo per la combattività ${ }^{16}$. Ronge stesso ammise in seguito che la crescente sfiducia dei soldati nei confronti della popolazione si estese anche ai cosiddetti "informatori confidenziali". Per questo motivo, persone che volevano dare informazioni a vantaggio dell'imperial-regio esercito venivano sottoposte a interrogatori sfiancanti al punto che le notizie degli informatori confidenziali avevano ormai perso ogni attualità ${ }^{17}$. Nelle aree di confine si creò perciò una forte incertezza nel distinguere tra amico e nemico. Oltre al sospetto di slealtà nei confronti dei propri connazionali, anche i prigionieri di guerra, finché si trovavano nelle immediate vicinanze delle aree di operazione, rappresentavano una grave minaccia alla sicurezza. Il loro rapido trasporto nella retroguardia era quindi nell'interesse dei comandi dell'esercito ${ }^{18}$. Tuttavia, alcune manchevolezze nella loro sorveglianza, soprattutto all'inizio della guerra, non erano rare $^{19}$.

Negli ultimi anni si può osservare un crescente interesse della ricerca storica per le tematiche connesse allo spionaggio, alle informazioni e ai servizi segreti ${ }^{20}$. Le riserve, che si potevano constatare soprattutto nel mondo accademico di lingua tedesca ${ }^{21}$, sono

\footnotetext{
${ }^{16}$ URBANSKI von Ostrymiecz, August, op. cit., p. 334.

${ }^{17}$ RONGE, Max, op. cit., p. 112. L'operato dell'esercito austro-ungarico e di altre autorità relativo al sospetto generalizzato di spionaggio nei confronti dei ruteni della Galizia, di parte degli slavi meridionali o nei confronti della popolazione italiana delle regioni meridionali e orientali, così come le situazioni di emergenza messe in atto appena prima della guerra che depotenziarono lo Stato di diritto sono già state affrontato dalla ricerca storica negli anni precedenti. Cfr.: LEIDINGER, Hannes, MORITZ, Verena, MOSER, Karin, DORNIK, Wolfram, Habsburgs schmutziger Krieg. Ermittlungen zur österreichisch-ungarischen Kriegsführung 1914-1918, St. Pölten-Salzburg-Wien, Residenz, 2014; SCHEER, Tamara, Die Ringstraßenfront. ÖsterreichUngarn, das Kriegsüberwachungsamt und der Ausnahmezustand während des Ersten Weltkrieges, Wien, Bundesministerium für Landesverteidigung und Sport, 2010; HOFFMANN, Georg, GOLL, Nicole-Melanie, LESIAK, Philipp: Thalerhof 1914-1936. Die Geschichte eines vergessenen Lagers und seiner Opfer, Herne, Schäfer, 2010.

${ }^{18}$ Dienstbuch J-35. Kriegsgefangenenwesen (Kgf. W.). Sammlung und Sichtung der ergangenen Erlässe, Wien 1915, p. 8.

${ }^{19}$ Cfr.: Militärkommando-Befehl, Nr. 22, Munkács, 23.9.1914, in: ÖStA/KA, NFA, Befehle, 11. Korps Lemberg, 1910-1915, Kt. 3.

${ }^{20}$ SCOTT, Len, JACKSON, Peter, «The Study of Intelligence in Theory and Practice», in Intelligence and National Security, 19, 2/2004, pp. 139-169.

${ }^{21}$ Cfr.: KRIEGER, Wolfgang, "German Intelligence History. A field in search of scholars», in Intelligence and National Security, 19, 2/2004, pp. 42-53; NEITZEL, Sönke, Neitzel, «Review of Beer, Siegfried, Martin Moll, Journal for Intelligence, Propaganda and Security Studies [JIPSS]. H-Soz-u-Kult», in H-Net Reviews, November 2009, URL: < http://www.hnet.org/reviews/showrev.php?id=26284 > [consultato il 3 ottobre 2016]. Siegfried Beer dello Austrian Center for Intelligence, Propaganda and Security Studies presso l'Università di Graz attribuisce a questa ricerca un salto in avanti delle scienze umane anglosassoni e scandinave
} 
state evidentemente accantonate ${ }^{22}$. Questo vale anche per la storiografia sullo spionaggio nella Prima guerra mondiale ${ }^{23}$. Si fanno, tuttavia, ancora attendere importanti progressi nella ricerca in relazione alle questioni dei servizi segreti e dello spionaggio nell'impero austroungarico durante il primo conflitto mondiale ${ }^{24}$. Le misure che sono state prese tra il 1914 e il 1918 nei confronti dei soldati dei diversi eserciti contrapposti sono ugualmente state poco indagate. Simili considerazioni possono essere fatte per questioni come l'effettiva efficacia delle informazioni provenienti dai prigionieri che venivano raccolte dai servizi segreti25. Mentre il comportamento delle autorità militari austroungariche nei confronti dei rischi di sicurezza relativi ai rimpatrianti dalla Russia in seguito alla rivoluzione d'Ottobre ha attratto per decenni l'attenzione dei ricercatori ${ }^{26}$, altri aspetti di questa problematica sono finiti in secondo piano. Queste sfaccettature riguardano, tra le altre cose, la questione: fino a che punto i rimpatrianti, ampiamente considerati pregiudizievolmente portatori di idee bolsceviche, sono stati considerati fonti di informazioni da parte dei servizi segreti austroungarici? L'articolo vuole affrontare le questioni relative ai soldati russi sotto prigionia austroungarica e ai soldati imperial-regi prigionieri in Russia. Questa intende

sugli studi sullo spionaggio. Cfr.: URL: < http://www.acipss.org/old/information/index.htm > [consultato il 3 ottobre 2016].

${ }^{22}$ A titolo di esempio deve essere citato il progetto "Kulturen der Intelligence" diretto da Sönke Neitzel, Philipp Gassert e Andreas Gestrich. Si veda, tra gli altri, URL: < https://www.gerdahenkel-stiftung.de/?page_id=8059s $>$ [consultato il 1 aprile 2016]. Una prospettiva storicoculturale viene anche ribadita da Robert Radu nella sua ottima introduzione ad un volume collettaneo sui servizi segreti pubblicato nel 2015: RADU, Robert, Spionage, Geheimhaltung und Öffentlichkeit - Ein Spannungsfeld der Moderne, in MEDROW, Lisa, MÜNZNER, Daniel, RADU, Robert, Kampf um Wissen. Spionage, Geheimhaltung und Öffentlichkeit 1870-1940, Paderborn, Ferdinand Schöningh, 2015, pp. 9-30. Cfr. anche: MORITZ, Verena, Zwei Imperien und ihr geheimes Wissen voneinander. Überlegungen zu einer Geschichte der militärischen Nachrichtendienste in Österreich-Ungarn und in Russland in den Jahren vor dem Ersten Weltkrieg, in Quaestion Rossica, III, 3/2005, pp. 78-97, pp. 81-83.

${ }^{23}$ Vedi: LARSEN, Daniel, Intelligence in the First World War: The State of the Field, in Intelligence and National Security, 29, 2/2014, pp. 282-302. Non viene esaminata approfonditamente l'ampia produzione storiografica russa. Da notare qui, è soprattutto: ALEKSEEV, Michail, Voennaja Razvedka Rossii. Pervaja Mirovaja Vojna, vol. 3, t. II, Čast'Pervaja i Vtoraja, Moskva, Izdatel'skij Dom „, Russkaja Razvedka“,2001. Cfr. anche le voci "Spionage" e "Geheimdienst" sull'enciclopedia online 1914-1918. Da prendere come esempio per il suo carattere di visione generale è: DEBRUYNE, Emmanuel, Espionage, in 1914-1918-online. International Encyclopedia of the First World War, cit.

${ }^{24}$ Opera fondamentale per questo ambito di ricerca è ancora: PETHÖ, Albert, Agenten für den Doppeladler. Österreich-Ungarns Geheimer Dienst im Weltkrieg, Graz, Leopold Stocker Verlag, 1998 , pp. 77-82.

${ }_{25}$ PETHÖ, Albert, op. cit. ;HUTTERER, Christine, Der Nachrichtendienst des k.u.k. Armeeoberkommando von 1914-1918 mit besonderer Berücksichtigung der italienischen Kriegsgefangenen- und Überläuferaussagen an der Tiroler Front, Wien, Dissertation, 1970.

${ }^{26}$ PLASCHKA, Richard Georg/HASELSTEINER, Horst/SUPPAN, Arnold, Innere Front Militärassistenz, Widerstand und Umsturz in der Donaumonarchie 1918, 2 voll., Wien, Böhlau, 1974. La ricerca è accessibile online dal 2015, URL:

< http://www.degruyter.com.uaccess.univie.ac.at/viewbooktoc/product/439268 > [consultato il 18 dicembre 2016]. 
essere un'analisi almeno a grandi linee, dati i limiti imposti nella redazione di questo scritto, ed evidenziare il significato di questi "gruppi” per i servizi segreti e di informazione dell'imperial-regio esercito ${ }^{27}$. Il testo deve quindi essere considerato come un tentativo di rendere comprensibile il ruolo che i prigionieri hanno giocato in relazione ai servizi di informazione dell'esercito austro-ungarico. Basato soprattutto su fonti conservate nell'Archivio Militare di Vienna, questo studio vuole presentare le diverse sfaccettature della prigionia in rapporto con le informazioni rilevanti per l'intelligence militare. Verranno presi in considerazione, accanto agli interrogatori dei prigionieri, il ruolo degli stessi come lunga manus dei propri servizi segreti (ad esempio con compiti di sabotaggio), il loro impiego pianificato in "operazioni sotto copertura" (partecipazione ad azioni militari) e aspetti della prigionia e del rimpatrio che indussero i servizi segreti imperial-regi a mettere in atto diverse contromisure. Da ultimo, in coordinamento con altri uffici militari e civili, queste misure si rivolgevano a soldati austroungarici sospetti di infedeltà durante il regime di prigionia o alla paura dell'importazione del bolscevismo tramite i soldati rimpatriati dalla Russia. Un aspetto importante rivestì il ruolo dei prigionieri per quel che riguardò le informazioni sulla Russia rivoluzionaria. Al contrario, i servizi segreti si occuparono anche della questione della sicurezza interna ${ }^{28}$ assieme al comportamento dei prigionieri di guerra russi, soprattutto dopo le rivoluzioni di febbraio e, in particolare, d'ottobre.

\section{Prigionieri di guerra russi come potenziali spie e sabotatori}

Il trasporto dei prigionieri dal fronte alla retroguardia fu, soprattutto nei primi mesi di guerra, in linea di massima, caotico ${ }^{29}$. Il provvedimento in base al quale per «un rapido alleggerimento della zona di operazioni [...] grandi masse di prigionieri di guerra» dopo la loro cattura dovevano essere rapidamente trasferiti nella retroguardia ${ }^{30}$, non venne mai effettivamente messo in pratica. Questo era dovuto sia

\footnotetext{
${ }^{27}$ Con l'inizio della guerra a partire dall'ufficio prove venne costituito il servizio di informazioni dello Stato maggiore. L'ufficio prove rimase attivo in parallelo.

${ }^{28}$ Cfr.: DAASE, Christopher, «Die Historisierung der Sicherheit. Anmerkungen zur historischen Sicherheitsforschung aus politikwissenschaftlicher Sicht», in Geschichte und Gesellschaft, 38, 3/2012, pp. 387-405.

${ }^{29}$ Questo caos nei trasporti favorì anche la trasmissione di malattie, poiché molti prigionieri erano già malati di tifo o colera quando erano stati catturati. Cfr.: DIETRICH, Elisabeth, Der andere Tod. Seuchen, Volkskrankheiten und Gesundheitswesen im Ersten Weltkrieg, in EISTERER, Klaus, STEININGER, Rolf Steininger (ed.), Tirol und der Erste Weltkrieg, Innsbruck, Studienverlag, 1995, p. 255-275, 256-258.

30 Dienstbuch J-35. Kriegsgefangenenwesen (Kgf. W.). Sammlung und Sichtung der ergangenen Erlässe, Wien, Druckerei des k. u. k. Kriegsministeriums, 1915, p. 8. Sulla costruzione del campo si veda: WALLECZEK, Julia, Hinter Stacheldraht. Die
} 
alla carenza di trasporti, sia a problemi logistici di natura generale. La priorità fu data ad un rapido interrogatorio dei prigionieri appena arrivati che, di regola, doveva avvenire entro tre giorni dalla cattura. "Coloro che venivano condotti dal fronte venivano portati il più velocemente possibile, sotto scorta, alla più vicina postazione dedicata che molto spesso si trovava presso il comando di corpo e, successivamente, presso il comando di divisione $o$, talvolta, anche a livelli più bassi» ${ }^{31}$. L'interrogatorio dei prigionieri non seguiva inizialmente alcun modello predeterminato in base al quale carpire le informazioni sul nemico che si sarebbero dovute ottenere. Si improvvisava. Secondo Maximilian Ronge questo durò fino alla fine di settembre del 1914, quando l'interrogatorio dei prigionieri venne adeguatamente sistematizzato ${ }^{32}$. Si può pensare che data l'importanza che il servizio di informazioni attribuiva alle testimonianze dei prigionieri, valesse evidentemente la pena di portare avanti una sorta di professionalizzazione di questa modalità di raccolta delle informazioni.

L’influenza diretta delle informazioni di intelligence sul nemico33 nelle decisioni politiche e militari concrete è, di regola, difficile da valutare in prospettiva storica ${ }^{34}$. Ciò non di meno, le valutazioni di personalità dotate di una indubbia autorità, indicano l'enorme significato che gli interrogatori dei prigionieri avevano per i militari. Il Generale Maggiore russo Pavel Fedorovič Rjabikov, attorno al 1919, riteneva, nella sua dissertazione sui Servizi segreti in tempo di guerra, che le informazioni ottenute interrogando i prigionieri rappresentarono una importante fonte di informazioni per la Razvedka durante la prima guerra mondiale. 35 Sul fronte avversario non si sosteneva diversamente. Maximilian Ronge scrisse, nel suo libro sullo spionaggio bellico e industriale nella Prima guerra mondiale, pubblicato nel 1930 e tenuto in alta

Kriegsgefangenenlager in den Kronländern Oberösterreich und Salzburg im Ersten Weltkrieg, Phil. Diss, Innsbruck, 2012, pp. 67-72.

${ }^{31}$ PETHÖ, Albert, op. cit., p. 77.

${ }^{32}$ RONGE, Max, op. cit., p. 112. Nel corso della guerra vennero inoltre elaborate delle istruzioni precise relative al trasporto dei prigionieri verso la retroguardia. Vedi: Verordnung des k.u.k. AOK, Chef des Generalstabs, betr. Abschub neu eingebrachter Kriegsgefangener im Falle einer eigenen Offensive, 25.8.1918, in ÖStA, KA, NL Ronge, B: 126: 2.

${ }^{33}$ Sui fondamenti e la trasmissione di notizie segrete si veda l'istruttiva trattazione di HORN, Eva, Der geheime Krieg. Verrat, Spionage und moderne Fiktion, Frankfurt am Main, Fischer Taschenbuch Verlag, 2007, pp. 79-155.

${ }^{34}$ Sulla questione degli effetti concreti del materiale di intelligence sui processi decisionali si veda, tra gli altri: MENNING, Brjus, Nasledie Agent Nr. 25, in Родина, 8, 2014, pp. 32-35; MENNING, Bruce, Russian Military Intelligence, July 1914: What St. Petersburg Perceived and Why It Mattered, in The Historian, 77, 2/2015, pp. 213-268. In relazione a questo si veda anche: MENNING, Bruce W., War Planning and Initial Operations in the Russian Context, in HAMILTON, Richard F., HERWIG, Holger H. (eds.), War Planning 1914, Cambridge, Cambridge University Press, 2010, pp. 80-142.

${ }^{35}$ Cfr. General-Maior Rjabikov, Razvedyvatel'naja služba v voennoe vremja, Tomsk, s.e., 1919, in ZDANOVIČ, Aleksandr Aleksandrovič, Antologija istorii specslužb. Rossija 1905-1924, Moskva, Kučkovo pole, 2007, pp. 291-410, p. 311. 
considerazione: «Per una gran parte delle nostre informazioni sul nemico dobbiamo ringraziare le dichiarazioni dei prigionieri di guerra che, durante alcuni periodi, in alcuni settori del fronte costituivano le uniche fonti di informazioni. Che russi, serbi e italiani trovassero dei partner tra gli irredentisti che erano tra i prigionieri di guerra austro-ungarici può sembrare ovvio, ma non altrettanto che anche noi potessimo fare grande uso dei prigionieri nemici» ${ }^{6}$. Durante gli interrogatori, secondo Ronge, non venne fatta alcuna pressione: «Alla fine i prigionieri di guerra erano in genere così tanti, che non era stata data indicazione di intrattenersi troppo a lungo con quelli che non collaboravano»37. È interessante osservare che agli organi deputati ad interrogare i prigionieri venne suggerito «di non interrogare sempre i più intelligenti [tra i prigionieri]» ${ }^{8}$. Nonostante tutto, secondo Ronge, anche gli ufficiali si dimostravano piuttosto loquaci non appena gli «fossero messe in vista» delle sigarette o gli venisse offerto un bicchiere di vino39. Una spiegazione più sottile della loquacità dei prigionieri potrebbe essere, in queste circostanze, che subito dopo la cattura la portata degli eventi per il proprio destino era difficile da valutare e, in particolare dopo un conflitto a fuoco sanguinoso, prevaleva la sensazione di sollievo per essere sopravvissuti.4o

La curiosità degli addetti agli interrogatori riguardava soprattutto la composizione e la forza di quelle unità di cui i soldati e ufficiali prigionieri avevano fatto parte, le prevedibili future operazioni del nemico, la tipologia e la composizione dell'armamento delle truppe nemiche, la quantità e qualità del vitto e degli approvvigionamenti, le vie di rifornimento oppure la domanda su quanto l'efficacia delle proprie armi venisse presa sul serio dal nemico41. Dai materiali conservati nell'Archivio Militare dell'Archivio di Stato Austriaco nel settore dei Neue Feldakten si può desumere che da parte degli uffici di ascolto non si utilizzassero dei questionari stampati. Talvolta dovevano bastare dei foglietti per appunti per trascrivere i risultati di un interrogatorio. Dopo il primo interrogatorio dei prigionieri da parte degli ufficiali addetti, ve ne era uno ulteriore «nel quale i dati personali venivano verificati e si avevano già a disposizione i protocolli del

\footnotetext{
${ }^{36}$ RONGE, Max, op. cit., p. 385 .

37 Ibidem.

${ }^{38}$ Bogen für Gefangenenbefragung, in ÖStA, KA, NL Ronge B: 126:2.

${ }^{39}$ RONGE, Max, op. cit., p. 385.

${ }^{40}$ Sulle specifiche condizioni degli imprigionamenti e sulle motivazioni per cui ci si consegnava si veda: GRAUER, Ryan, «Why Do Soldiers Give Up? A Self-Preservation Theory of Surrender», in Security Studies, 23, 3/2014, pp. 622-655. Si vedano in particolare le pp. 636-649.

${ }^{41}$ Cfr. ad esempio un questionario dell'anno 1916 allegato a: HUTTERER, Christine, Der Nachrichtendienst des k.u.k. Armeeoberkommando von 1914-1918 mit besonderer Berücksichtigung der italienischen Kriegsgefangenen- und Überläuferaussagen an der Tïroler Front, Wien, Dissertation, 1970.
} 
primo interrogatorio nei quali venivano annotate delle integrazioni» ${ }^{42}$. Solo dopo questa procedura avveniva il trasporto nei centri di raccolta dai quali veniva gestita l'ulteriore permanenza dei prigionieri. Che le «apparecchiature per l'ascolto delle conversazioni»43 citate da Maximilian Ronge venissero utilizzate in questi campi di raccolta o nei campi stabili nella retroguardia non è ancora stato stabilito. È un dato di fatto che i soldati venivano messi in guardia, in caso di cattura, sul non discutere di cose militari nei campi: «Non dimenticare che nei campi di prigionia si aggirano informatori travestiti che origliano ogni conversazione e che i nostri ufficiali vengono spiati»44. Gli ufficiali del servizio informazioni sapevano ciò che dicevano. In effetti anche loro cercavano di infiltrare degli informatori nei campi di prigionia della monarchia. A partire dal 1917 i rapporti di questi informatori confidenziali divennero sempre più importanti, poiché ci si voleva fare un quadro delle idee politiche dei prigionieri nei confronti degli eventi rivoluzionari occorsi nella loro madrepatria45.

Uno dei compiti più importanti dei punti di ascolto consisteva tuttavia nella valutazione delle informazioni raccolte attraverso gli interrogatori dei prigionieri. In modo particolare, si trattava di confrontare tra loro i risultati degli interrogatori. Se le dichiarazioni collimavano nei punti essenziali, allora le informazioni venivano valutate come attendibili. I dati a disposizione dei punti di ascolto potevano in questo modo essere verificati o meno ${ }^{46}$. Inoltre, venivano distinte le informazioni ottenute da soldati che erano stati regolarmente imprigionati da quelli che si erano consegnati. Le dichiarazioni di questi ultimi venivano considerate particolarmente importanti poiché in alcune situazioni si poteva verificare una maggiore propensione alla condivisione di informazioni 4 .

I disertori, come anche i militari che finivano regolarmente in prigionia, fornivano agli ufficiali di ascolto dell'imperial-regio esercito, tra le altre cose, informazioni che riguardavano il morale delle truppe nemiche e davano anche indicazioni riguardo al fatto che tra i soldati vi fossero già gruppi numerosi che si erano accordati fra di loro

\footnotetext{
${ }^{42}$ PETHÖ, Albert, op. cit., pp. 77-78.

${ }^{43}$ RONGE, Max, op. cit., p. 385; PETHÖ, Albert, op. cit., p. 78

${ }^{44}$ Kommando der k.u.k. Isonzo-Armee (Glstbs.-Abt.), Op. Nr. 6300: Vorschrift für die Geheimhaltung, in ÖStA, KA, AOK Evidenzbüro, Kt. 3711, p. 14.

45 Schreiben des k.u.k. Kriegsministeriums, Abt. 10. Kgf. betreffend Behandlung der Konfidentenangebote Kriegsgefangener, 27.4.1918, in ÖStA, KA, 10. Abt. Kgf. 1918, Kt. 2008, ohne Zahl.

${ }^{46}$ I compiti dei punti di ascolto erano numerosi e includevano, tra le altre cose, l'osservazione aerea tramite palloni, le intercettazioni telefoniche, la ricognizione aerea, la cosiddetta fotografia a distanza e anche l'analisi di oggetti e documenti requisiti al nemico. Cfr.: HUTTERER, Christine, op. cit., pp. 22-23.

${ }^{47}$ Cfr.: Kommando der k.u.k. Isonzo-Armee (Glstbs.-Abt.), Op. Nr. 6300: Vorschrift für die Geheimhaltung, in: ÖStA, KA, AOK Evidenzbüro, Kt. 3711, p. 13.
} 
per consegnarsi al nemico al prossimo scontro a fuoco ${ }^{48}$. Venivano anche chieste le ragioni che vi erano dietro ad una volontà combattiva che spesso veniva indicata come altalenante. I prigionieri russi affermavano, per esempio, che il trattamento duro da parte degli ufficiali zaristi o il mancato riconoscimento di particolari prestazioni alimentavano lo scoraggiamento dei soldati e avrebbero portato alla diserzione 49 .

Anche il morale nelle retrovie attirava l'interesse dei servizi di informazione. Questo riguardava anche informazioni analoghe provenienti dai servizi segreti dell'alleato tedesco. In uno scritto reindirizzato a Vienna da Berlino che riassumeva tutti i rapporti e le diverse testimonianze sulla situazione interna dell'impero zarista fino al maggio 1915, si sosteneva che con lo «scoppio della rivoluzione la fine della guerra» non poteva essere messa in conto ${ }^{50}$. Le autorità dell'imperial-regio esercito raccolsero ovviamente importanti informazioni di carattere militare e sulla situazione in Russia anche tramite la verifica della corrispondenza dei prigionieri51. Già fin dall'inizio del 1915 furono censurate circa un milione di lettere. Solo pochi mesi dopo l'ufficio di censura, che collaborava strettamente con l'ufficio informazioni, ebbe a che fare settimanalmente con circa un milione di lettere ${ }^{2}$.

Già pochi mesi dopo lo scoppio della guerra le autorità dell'imperial-regio esercito iniziarono ad utilizzare prigionieri di guerra in grande quantità per diversi lavori al di fuori dei campi di prigionia. Progressivamente persero importanza i campi, nei quali rimanevano solamente ufficiali, che secondo la Convenzione di guerra de L'Aia non potevano essere costretti al lavoro, o prigionieri malati. Crescevano continuamente le sfide nell'ambito della sorveglianza e del controllo dei prigionieri di guerra. Nell'ottica di un'evidente e crescente carenza di manodopera nella Doppia monarchia, le autorità militari austriache si convinsero alla fine ad affidare ai datori di lavoro contingenti di oltre 200 prigionieri. Mentre i prigionieri che, ad esempio, lavoravano in grandi gruppi nelle fabbriche, venivano ospitati in buona parte in baraccamenti costruiti da essi stessi, la sorveglianza dei soldati nemici che venivano impiegati anche isolatamente in diversi luoghi di lavoro divenne in qualche modo difficile. Mancava, per di più,

\footnotetext{
${ }^{48}$ Raccoglitore con testimonianze di prigionieri del 31.1. 1916, in ÖStA, KA, NFA, 8. Korps, Kt 1087 Grpkmdo. Fzm. Benigni 1916 K.-Akten, Kt. 1087.

${ }^{49}$ Ibidem

${ }^{50}$ Deutscher Bericht über die Stimmung in Russland vom 19.5.1915, in: ÖStA, KA, AOK Evidenzbüro, Kt. 3711.

${ }^{51}$ L'analisi della corrispondenza dei prigionieri "a scopo di raccolta informazioni“ diede, secondo il comando austriaco, i migliori risultati: Schreiben des k.u.k. AOK an den Chef des Ersatzwesens vom 17.9.1917, in ÖStA, KA, Chef d. E. W. 1917: 19-40, Kt. 42.

${ }^{52}$ PETHÖ, Albert, op. cit., p. 78.
} 
l'adeguato personale di guardia53. Inoltre, l'impiego di prigionieri di guerra russi in aree della monarchia a maggioranza slava veniva collegato col pericolo di una potenziale "fraternizzazione". 54

In generale, la crescente decentralizzazione che andava di pari passo con il prolungamento della guerra produsse una perdita di controllo che le autorità di sicurezza vedevano come una minaccia. Questo avvenne anche a causa di una situazione degli approvvigionamenti in drammatico peggioramento a partire dal 1916, soprattutto nelle parti occidentali della monarchia danubiana e con essa un calo di disciplina da parte dei prigionieri. La crisi alimentare minacciava inoltre la propaganda politica tra i prigionieri che appartenevano a diverse nazionalità dell'impero zarista e avrebbero potuto essere motivati alla lotta anti-russa. La non trascurabile offerta di privilegi legati al sostentamento per questi prigionieri divenne difficile da sostenere alla luce della situazione precaria degli approvvigionamenti nell'impero asburgico55.

Mentre nel frattempo gli sforzi dei russi per creare formazioni di soldati italiani e slavi dell'imperial-regio esercito da impiegare contro l'Austria-Ungheria proseguivano con una certa veemenza e le notizie di ciò arrivavano ai comandi delle armate imperiali, da parte austro-ungarica simili sforzi erano caratterizzati da grande cautela. Un'operazione militare degli imperi centrali contro la Russia che coinvolgesse il servizio di informazioni austro-ungarico e che vedesse la partecipazione di prigionieri di guerra ucraini non venne messa in atto. Il trasferimento di prigionieri all'esercito turco alleato o da indirizzare alle legioni polacche e ucraine rimase limitato numericamente e avvenne solo dopo numerosi scambi di opinioni o rimase un fenomeno temporaneo56. L'esortazione esplicita al "tradimento" nei confronti dei soldati nemici fu dibattuta anche nei volantini che da parte austro-ungarica venivano lanciati su alcuni settori del fronte. Simili formule venivano, in definitiva, evitate, in questi scritti propagandistici. In questi appelli si incitavano i soldati, senza possibilità di equivoco, a disertare e a consegnare le armi. Nel 1916 la propaganda imperial-regia prometteva, in diversi settori del fronte, a chi si fosse consegnato con le armi in mano, 5

\footnotetext{
${ }^{53}$ Cfr.: «Mangelhafte Bewachung der Kriegsgefangenen», in Linzer Volksblatt, 12 febbraio 1918, p.4.

${ }_{54}$ MORITZ, Verena, Zwischen Nutzen und Bedrohung, cit., pp. 109-115.

${ }_{55}^{55}$ MORITZ, Verena, Zwischen Nutzen und Bedrohung, cit., pp. 329-330.

56 BIHL, Wolfdieter, «Das im Herbst 1914 geplante Schwarzmeer-Unternehmen der Mittelmächte», in Jahrbücher für Geschichte Osteuropas, Neue Folge, XIV, 3/1966, pp. 326366; MORITZ, Verena, Zwischen Nutzen und Bedrohung, cit., pp. 329-330.
} 
rubli e un copeco per ogni proiettile. A partire dal 1917 venivano offerte dieci corone per la consegna dell'arma57.

Già nel 1916, ossia prima delle rivoluzioni di febbraio e ottobre del 1917, la propensione, in particolare dei prigionieri russi, ad abbandonare il le proprie attività lavorative era vistosamente elevata. I motivi di ciò erano diversi, dal punto di vista dei comandi militari imperial-regi, e andavano dalle già citate proteste per la pessima situazione alimentare associata ad un lavoro sempre più duro, fino all'idea di indebolire il nemico con scioperi e sabotaggi per abbreviare il conflitto. Soprattutto i prigionieri nuovi arrivati - così pensavano i militari austro-ungarici - erano stato istruiti dai loro superiori per agire in questo modo e contro gli interessi degli Stati nemici in caso di cattura $^{58}$. Nel 1917 la notizia di un sabotaggio compiuto da prigionieri arrivò alla stampa. Ad alcuni prigionieri erano state effettivamente rinvenute delle istruzioni per il sabotaggio che facevano riferimento all'incendio doloso $o$, ad esempio, al danneggiamento dell'agricoltura tramite la distruzione delle sementi. In considerazione del fatto che centinaia di migliaia di prigionieri non solo lavoravano nelle aziende agricole ma anche nelle fabbriche di armi o con l'esercito al fronte nelle zone in cui si svolgevano le operazioni, questo tipo di atti di sabotaggio sembrava generare un pericolo tale da essere preso sul serio59. Solo poche settimane dopo i primi articoli di giornale di questo tenore, però, giunsero i primi segnali di distensione ${ }^{60}$. Dietro le quinte si restava tuttavia in allerta. Il ministero della guerra imperial-regio indirizzo nel giugno 1918 una richiesta dei servizi d'informazione ai campi di prigionia della monarchia, nella quale venivano richieste relazioni precise su qualsivoglia atto di spionaggio condotto da parte dei prigionieri. Alle persone a cui era indirizzata venne quindi spiegata l'ampia gamma di atti di spionaggio da osservare e inoltre, accanto allo spionaggio industriale si doveva tenere d'occhio anche il tentativo di portare avanti propaganda rivoluzionaria tra la popolazione locale ${ }^{61}$.

\footnotetext{
${ }^{57}$ Cfr. ASTAŠOV, Aleksandr Borisovič, Propaganda na Russkom fronte v gody Pervoj mirovoj vojny, Moskva, Speckniga, 2012, pp. 105-106; cfr. anche un volantino con l'appello alla consegna delle armi riprodotto nell'appendice di: HUTTERER, Christine, op. cit.

${ }^{58}$ Questa opinione deriva dai materiali archivistici analizzati nell'ambito del già citato progetto sui prigionieri di guerra nell'impero asburgico. Cfr. ad esempio: Wahrnehmungen hinsichtlich neu eingebrachter Kriegsgefangener, August 1916, in ÖStA, KA, KM 10. Abt. Kgf. 1916: 10-147/2. Per combattere gli scioperi die prigionieri vennero create delle apposite pattuglie antisciopero. Cfr.: Schreiben der Kgf.-Abt. vom April 1018, in ÖStA, KA KM 10. Abt. Kgf. 1918: 147-10/35.

${ }^{59}$ Cfr. Wiener Zeitung, 17 maggio 1917, pp. 7-8.

${ }^{60}$ Cfr. Fremden-Blatt, 11 agosto 1917, p. 6.

${ }^{61}$ Schreiben des Kriegsministeriums betreffend Spionageversuche von Kriegsgefangenen, 15.6.1918, in ÖStA, KA, KM 10. Abt. Kgf. 1918: 10-4/4.
} 


\section{Prigionieri austro-ungarici in Russia}

Tanto più i servizi d'informazione austro-ungarici si impegnavano per ottenere informazioni sulle forze armate nemiche dai prigionieri di guerra, tanto meno erano preoccupati del fatto che i propri soldati potessero cadere prigionieri o disertare e, allo stesso modo, fornire informazioni sul proprio esercito. Quando divenne chiaro che, come conseguenza di grandi manovre di accerchiamento, la prigionia era un destino di massa $^{62}$, l'amministrazione militare austro-ungarica prese le distanze, almeno parzialmente, dall'idea generalmente negativa sulla prigionia che aveva dominato fino a quel momento. Perlomeno nella comunicazione verso l'esterno non veniva più trasmessa l'opinione che la cattura di centinaia di migliaia di soldati austro-ungarici fosse da attribuire esclusivamente ad una loro responsabilità o tantomeno che questi dovessero essere generalmente identificati come potenziali traditori. Tuttavia, la leadership militare austro-ungarica manteneva una posizione ambivalente nei confronti della prigionia ${ }^{63}$. Gli ufficiali austro-ungarici che finivano in prigionia senza essere feriti dovettero, al loro ritorno, essere sottoposti a processi disciplinari. Inoltre, già dall'inizio del conflitto, il comportamento dei soldati slavi veniva osservato con una certa diffidenza. Le notizie sulla diserzione di soldati cechi o le informazioni sull'adesione della maggioranza dei prigionieri slavi alle legioni (Druscine) in Russia, raggiunsero ben presto gli ufficiali addetti agli interrogatori dei prigionieri. Già nel 1915 le domande ai prigionieri e ai disertori relative alla Legione ceca (antesignana della Legione cecoslovacca successivamente creata dall'esercito russo ndt) erano piuttosto comuni. Gli sforzi dei servizi segreti legati alla verifica del comportamento sleale dei soldati austro-ungarici in prigionia ebbero come conseguenza un significativo incremento della documentazione ${ }^{64}$. Nel frattempo i soldati venivano istruiti, in caso di prigionia, a limitarsi a dichiarare i propri dati personali. Ad essi veniva reso noto che, se non si fossero attenuti a questa regola comportamentale, ne avrebbero dovuto rispondere a guerra finita ${ }^{65}$. Nonostante questo è piuttosto ovvio che le pratiche standard di interrogazione dei prigionieri in Russia includessero domande di carattere

\footnotetext{
${ }^{62}$ RACHAMIMOV, Alon, POWS and the Great War: Captivity on the Eastern Front, Oxford, Berg, 2002, p. 38.

${ }_{63}$ MORITZ, Verena, LEIDINGER, Hannes, Der Sinn der Erfahrung. Gedanken über den Umgang mit Selbstzeugnissen ehemaliger Kriegsgefangener des Ersten Weltkrieg, in LEIDINGER, Hannes, MORITZ, Verena Moritz (Hg.), In russischer Gefangenschaft. Erlebnisse österreichischer Soldaten im Ersten Weltkrieg, Wien-Köln-Weimar, Böhlau, 2008. pp. 18-19.

${ }^{64}$ La documentazione in merito si trova accanto agli atti dell'Ufficio prove e in gran quantità nei fondi del ministero della guerra e nei fondi denominati Neue Feldakten.

${ }^{65}$ HUTTERER, Christine, op. cit., pp. 172-173.
} 
militare sia per i nemici catturati, sia per coloro che rimpatriavano dalla prigionia austro-ungarica ${ }^{66}$.

Parallelamente alle istruzioni sul comportamento da tenere in prigionia per i soldati austro-ungarici, ai comandi militari imperial-regi venne ordinato di mostrarsi senza pietà nei confronti di potenziali traditori. La «diserzione» non doveva essere tollerata «in nessuna circostanza». Un ordine relativo a questo tema recitava: «Chiunque se ne renda responsabile, non importa se soldato od ufficiale, [è da] fucilare»67. Nelle istruzioni interne all'esercito veniva sempre ribadito quanto pericolose fossero considerate le informazioni in possesso di soldati austro-ungarici finiti in prigionia ${ }^{68}$.

La prigionia doveva però anche essere utilizzata per condurre operazioni sotto copertura. Sono ormai finite nell'oblio le attività di spionaggio dell'ufficiale imperialregio Roland Strunk, che a causa della loro versione romanzata apparsa nel 1938 furono collocate nell'ambito della leggenda69. $\mathrm{Fu}$ un ardente sostenitore del nazionalsocialismo, Herbert Volck, che nel 1938 mise su carta la vita di Roland Strunk sulla base di una sinossi fornita di prima mano dallo stesso ufficiale. Che queste memorie fossero apparse nel contesto dell'annessione dell'Austria al Terzo Reich è da considerarsi uno dei motivi che hanno fatto sì che dopo il 1945 il tema dello spionaggio austro-ungarico non fosse più preso in considerazione ${ }^{70}$. Nonostante una verifica complessiva delle affermazioni di Volck risulti difficile, data la mancanza del materiale d'archivio dei servizi d'informazione austro-ungarici a causa della loro sistematica distruzione nell'autunno del 191871, l'attività di Strunk può comunque essere a grandi linee considerata attendibile ${ }^{72}$. Il giovane ufficiale si fece prendere prigioniero di proposito (almeno secondo la versione narrata da Volck), per poter minare un importante tratto ferroviario in Russia. In questo modo si sarebbero dovute

\footnotetext{
${ }^{66}$ Cfr. ad esempio: RGVIA (Rossijskij Gosudarstvennyj Voenno-Istoričeskij Archiv), Fond 2048 (Štab glavnokomandujuščego armijami Zapadnogo fronta), op. 1, dela 509, 510, 511, 512. I documenti sono stati visti a Mosca, nel 2015 all'inizio del già citato progetto di ricerca.

${ }^{67}$ Vorschrift für die Geheimhaltung, in ÖStA, KA, AOK Evidenzbüro, Kt. 3711, p. 13.

68 Der militärische Abwehrdienst (Organisation, Weisungen für die Durchführung des militärischen Abwehrdienstes und für die Mitwirkung bei der Nachrichtenerbringung), in ÖStA, KA, AOK Evidenzbüro, Kt. 3711, p. 21.

${ }^{69}$ VOLCK, Herbert, Der Traum vom Tode. Das phantastische Leben des berühmten deutschen Weltreporters Roland Strunk, Berlin, Holle \& Co. Verlag, 1938.

${ }^{70}$ Volck, che fu attivo nei servizi di informazione durante il regime nazionalsocialista, cadde comunque in disgrazia presso gli ambienti nazisti e nel 1944 fu giustiziato a Buchenwald. Cfr. URL: < http://www.polunbi.de/pers/volck-01.html > [consultato l'8 agosto 2016].

${ }^{71}$ MORITZ, Verena, LEIDINGER, Hannes, Im Zentrum der Macht, cit., pp. 171-172.

${ }^{72}$ Nei fondi conservati presso il Kriegsarchiv e che riguardano i servizi segreti militari, non si trovano tracce di Roland Strunk. Al contrario, nei materiali dell'archivio militare di Mosca e che riguardano i piani relativi ai prigionieri degli ambienti militari di Irkutsk, si trovano tracce che riguardano l'arresto di Strunk per spionaggio. Sulla morte di Strunk in seguito ad un duello si veda: Das Interessante Blatt, 28 ottobre 1937, p. 4.
} 
interrompere le linee di rifornimento per il fronte. Strunk, che agiva per conto del servizio d'informazioni austro-ungarico, venne tuttavia catturato prima di poter portare a termine il suo piano. Venne condannato a morte, ma riuscì a scappare e, nel 1918, a rientrare in patria73. Dopo la guerra si trasferì in Germania e si unì ai nazionalsocialisti. In seguito divenne corrispondente di guerra per il «Völkischer Beobachter »e, nel 1937, perì in modo violento: in carica come ufficiale delle SS, morì per le gravi ferite che si era procurato durante un duello 74

Come molti degli osservatori di quegli eventi, Strunk, che visse entrambe le rivoluzioni russe del 1917, associava gli sconvolgimenti che colpirono i territori dell'ex impero dei Romanov con il caos e il declino. Ritornò in patria convinto di un forte sentimento antibolscevico. In considerazione della confusa situazione in Russia, è difficile trarre delle informazioni affidabili sulla situazione dei prigionieri austroungarici e tantomeno sugli effettivi sviluppi che avvennero nell'ex impero zarista tra il 1917 e il 1918. "Occhi e orecchi" del servizio informazioni austro-ungarico divennero le delegazioni autorizzate nell'ottica delle trattative di pace tra le potenze centrali e il governo sovietico e le cosiddette Kriegsgefangenenexposituren, unità amministrative che dovevano organizzare il ritorno dei prigionieri dalla Russia. Gli ufficiali distaccati in queste strutture provvedevano, con le loro relazioni, a fornire ai servizi d'informazione un sguardo d'insieme sugli eventi che accadevano in luogo.

Mentre il governo rivoluzionario guidato da Lenin concluse a Brest-Litovsk una pace che venne molto discussa anche in seno ai bolscevichi, a Vienna e Berlino, come anche a Londra, Parigi, Washington e Tokyo, si pensava in termini di prosecuzione della guerra mondiale. Da questi pensieri la Russia non venne esclusa come terreno strategico come non vennero esclusi i soldati delle potenze centrali che vi si trovavano. Questi ultimi venivano percepiti dalle potenze dell'Intesa come una minaccia, sia per le notizie sull'«influenza bolscevica» a cui erano esposti, sia perché il loro reclutamento nella nascente Armata Rossa veniva considerata come una manovra dei vertici militari tedeschi. Poiché Lenin, secondo l'opinione delle autorità in Francia e Gran Bretagna era considerato un "agente" tedesco, si vedeva quindi nel riarmo dei soldati tedeschi, ungheresi e austriaci nei territori dell'ex impero dei Romanov - che in realtà veniva fortemente contrastato dall'Austria-Ungheria e dalla Germania, perlomeno in diretto collegamento con un supporto ai bolscevichi - una sorta di grande piano degli imperi

\footnotetext{
${ }^{73}$ Cfr. «Kurzmitteilung», in Neue Freie Presse, 19 aprile 1918, p. 6.

${ }^{74}$ Cfr. «Kurzmitteilung», in Das Interessante Blatt, 28 ottobre 1937, p. 4.
} 
centrali. Il Giappone e le potenze occidentali non davano poca importanza a questo tipo di considerazioni e propesero per un intervento militare in Russia75.

Più chiari furono gli effetti generati dall'ingresso massiccio, nel febbraio 1918, della Legione cecoslovacca costituita da ex prigionieri di guerra che si trovavano nelle regioni del Volga, degli Urali e della Siberia. Dopo la caduta dello Zar nel febbraio del 1917 questa formazione vide crescere in modo sostanzioso la propria consistenza ${ }^{76}$. Per il governo di Lenin questi sviluppi non rimasero senza conseguenze. Ai fianchi e alle spalle della legione dei volontari cecoslovacchi si organizzò l'opposizione russa contro i bolscevichi. Al tempo stesso il presidente americano Woodrow Wilson, finora tentennante, decise di dare il suo consenso ad una limitata azione militare americana in sostegno ai "druscinisti" 77.

Già prima che i sostenitori della politica interventista muovessero i loro passi, in alcune regioni la guerra tra sostenitori e avversari del regime del Partito comunista si manifestò come contrapposizione tra diverse nazionalità della monarchia danubiana. Ad esempio, i cechi "bianchi” si ponevano in contrasto con i "rossi": tedeschi, austriaci e soprattutto ungheresi. Che la guerra, soprattutto come conseguenza di conflitti etnici ed internazionali, non fosse più limitata alla periferia ma fosse ormai giunta nel cuore della Russia, si correlava strettamente col problema dei prigionieri di guerra. Per alcuni mesi l'intervento di internazionalisti, cioè di soldati rivoluzionari provenienti dalla Germania e soprattutto dall'Austria-Ungheria, fu di grande importanza per le forze armate sovietiche ${ }^{78}$. In molti casi le forze internazionaliste più affidabili venivano anche incaricati del «mantenimento dell'ordine interno»79. Non da ultimo, questi fenomeni, che mostravano la slealtà dei membri dell'armata imperial-regia, giunsero a conoscenza

75 Bericht des k.u.k. Kriegsministeriums bezüglich der alliierten Intervention und der Bewaffnung von Kriegsgefangenen in Russland, Mai/Juni 1918, in ÖStA, KA, KM 10. Abt. Kgf. 1918: 10-7/7-257; UNTERBERGER, Betty Miller, America's Siberian Expedition 1918-1920. A Study of National Policy, New York, Duke University Press, 1969, p. 47.

${ }^{76}$ KALVODA, Josef, Czech and Slovak Prisoners of War in Russia During the War and Revolution, in WILLIAMSON, Samuel R., PASTOR, Peter (eds.), Essays on World War I: Origins and Prisoners of War, New York, Columbia University Press, 1983, pp. 215-249, pp. $223-225$.

${ }_{77}$ UNTERBERGER, Betty Miller, The United States, Revolutionary Russia and the Rise of Czechoslovakia, Chapel Hill, University of North Carolina, 1989, p. 321.

78 LEIDINGER, Hannes, MORITZ Verena, Gefangenschaft, Revolution, Heimkehr. Die Bedeutung der Kriegsgefangenenproblematik für die Geschichte des Kommunismus in Mittelund Osteuropa 1917-1920, Wien-Köln-Weimar, Böhlau, 2003, pp. 387-409. Außerdem: Schreiben Ernst Reuters über die Anfänge rätefreundlicher Gefangenenorganisationen 1917/18, in RGASPI (Rossijskij Gosudarstvennyj Archiv Social'no-Političeskoj Istorii), f. 549, op. 1, d. 92.

${ }^{79}$ LEIDINGER, Hannes, MORITZ Verena, Gefangenschaft, Revolution, Heimkehr, cit., pp. 421439. Sulle valutazioni della leadership dell'esercito austro-ungarico a proposito del movimento internazionalista sulla base di testimonianze oculari e relazioni di ufficiali austro-ungarici vedasi: Bericht der k.u.k. Vertretung in der Ukraine an das Ministerium des Äußern, 15.6.1919, in ÖStA, HHStA, MdÄ, PA I, Kt. 1076, Zl. 6616, Fol. 259-26. 
dei servizi d'informazione austro-ungarici. Tra le altre cose, era fondamentale valutare la dimensione del tradimento. Nel frattempo veniva portato avanti il disfacimento dell' esercito imperial-regio: con i prigionieri rimpatrianti nell'impero come conseguenza dei trattati di Brest-Litovsk, il governo sovietico voleva trasmettere il "bacillo del bolscevismo" al di fuori della propria sfera d’influenza. I rimpatrianti dalla prigionia russa dovevano diventare «messaggeri della rivoluzione mondiale». A Vienna erano in allarme e pianificarono una sorta di quarantena ideologico-morale, per "rieducare" coloro che rientravano dalla Russia prima di reintegrarli nell'esercito e di trasportarli verso l'interno del paese. Queste misure erano connesse con interrogatori circostanziati dei reduci riguardo alle loro esperienze in Russia ${ }^{80}$. Si volevano ottenere informazioni sulla situazione in Russia. Anche le informazioni militari sulle operazioni, nonché sulla consistenza, delle forze militari bolsceviche e anti-bolsceviche giocavano un ruolo importante. Nel quadro degli interrogatori dei rimpatriati le misure per il mantenimento della sicurezza interna andavano di pari passo con l’intelligence militare.

Una parte fondamentale delle informazioni - che i servizi segreti organizzarono fino al collasso della monarchia danubiana in relazioni settimanali - provenivano, sin dalla rivoluzione di febbraio, dai rimpatriati dalla prigionia ${ }^{81}$. In generale gli sforzi dei servizi segreti in relazione alla Russia non sembrano semplicemente plasmati dall'interesse per i fatti che stavano avvenendo, ma anche dall'intenzione di sviluppare un'attività di intelligence di lungo periodo in Russia ${ }^{82}$. Era ancora fresco nella memoria il fatto che l'ufficio prove del ministero della Guerra, che prima del 1914 era protagonista della "guerra tra servizi segreti" con la Russia era stato messo in secondo piano $^{83}$. Si voleva allora cogliere l'occasione per creare nuove reti di spionaggio e porre le basi per futuri "centri di ascolto" in Russia contro di essa. I rimpatrianti dalla Russia

\footnotetext{
${ }^{80}$ LEIDINGER, Hannes, MORITZ Verena, Gefangenschaft, Revolution, Heimkehr, cit., pp. 453467.

${ }^{81}$ Questi rapporti si possono trovare, ad esempio, in: ÖstA, KA, Kdo d. SW-Front II, 1917: Russische Revolution Berichte, Kt. 677.

${ }_{82}$ Sul grande interesse delle dichiarazioni dei rimpatrianti per i servizi di informazioni, soprattutto nel momento in cui la pace tra gli imperi centrali e la Russia non era ancora stipulata, si veda anche: Protokoll einer Besprechung von Nachrichtendienstoffizieren der Militärkommanden und der Militärgouvernements Lublin und Belgrad, 25.8.1917, in: ÖStA, KA, NL Ronge B: 126:2.

${ }^{83}$ Le valutazioni sull'efficienza dei servizi segreti nell'impero asburgico e in Russia prima della guerra mondiale sono molto diversificate. Questo non vale solo per i servizi segreti in generale, bensì anche a proposito dei loro effetti nei confronti dei singoli Stati. Una ricerca approfondita in questo senso che faccia ricorso ai giudizi dei contemporanei e alle memorie successive sarebbe auspicabile. Cfr. su questo, ad esempio: DALY, Jonathan W., «Security Services in Imperial and Soviet Russia», in Kritika: Explorations in Russian and Eurasian History, 4, 4/2003, pp. 955-973; PETHÖ, Albert, op. cit., pp. 30-56, 236-238.
} 
erano particolarmente adatti per questo compito ${ }^{84}$, mentre l'arruolamento di prigionieri di guerra russi, che avrebbero dovuto fungere da informatori confidenziali per i servizi segreti austro-ungarici dopo il loro rimpatrio, sembrava meno sensato ${ }^{85}$. La nomina dell'ex capo del servizio infomazioni, Oskar von Hranilovic-Czvetassin, precedentemente un alto ufficiale dei servizi segreti, a futuro delegato militare in Russia, dimostra allo stesso modo il grande interesse di Vienna per un efficiente «servizio di informazioni sulla Russia» 86 .

Nel frattempo, il pregiudizio generalmente diffuso della leadership militare che considerava la gran parte dei prigionieri di ritorno come sospettati di tradimento e "infetti dal germe rivoluzionario" portò ad uno scontento tra i reparti ausiliari dell'armata asburgica che, fino alla metà del 1918, furono protagonisti di ammutinamenti in Stiria, Boemia settentrionale, Ungheria meridionale e Serbia. La gran parte dei rimpatrianti dalla Russia, tuttavia, non diede segno di ribellione in questa fase finale della monarchia danubiana e dopo il crollo della monarchia asburgica rimase passiva o diresse le sue attenzioni verso altre correnti politiche non comuniste ${ }^{87}$.

I combattimenti in Russia, che chiamarono in causa una grande varietà di gruppi con diversi obbiettivi politici e "visioni" nazionali, influenzarono al contempo anche lo scambio di prigionieri tra le due parti. Sulla base di informazioni di intelligence sulla situazione in Russia, a Vienna si rifletteva su quale peso avrebbero potuto avere $\mathrm{i}$ prigionieri russi che rientravano nel loro paese. Con l'intenzione di evitare l'apertura di un nuovo fronte a est dell'ex impero zarista e quindi l'ulteriore coinvolgimento delle forze dell'Intesa, secondo le indicazioni del Comando Supremo austriaco si sarebbero dovuti trattenere temporaneamente i prigionieri «potenzialmente favorevoli all'Intesa» e quelli abili al servizio militare provenienti dai territori a est e nord del Volga e dalla Russia asiatica. Al tempo stesso il Ministero della guerra si impegnò per il rapido rimpatrio di circa 70.000 prigionieri austro-ungarici. Per quanto riguardava il rimpatrio dei rimanenti prigionieri che si trovavano ancora negli ex territori zaristi, il Ministero della guerra aveva abbandonato le speranze. Un ritorno di questi soldati dalle zone di combattimento, dove forze russe e straniere, compresa la Legione cecoslovacca,

${ }^{84}$ Cfr.: RUTKOWSKI, Ernst, «Der Kundschaftsdienst des k.u.k. Armeeoberkommandos in Rußland im Jahr 1918», in Österreichische militärhistorische Forschungen, 1, 1992, pp. 15-78, p. 26.

${ }_{85}$ Protokoll einer Besprechung von Nachrichtendienstoffizieren der Militärkommanden und der Militärgouvernements Lublin und Belgrad, 25.8.1917, in: ÖStA, KA, NL Ronge B: 126:2.

${ }^{86}$ LEIDINGER, Hannes, MORITZ, Verena, Gefangenschaft, Revolution, Heimkehr, cit., p. 359.

${ }^{87}$ Cfr.: PLASCHKA, Richard Georg, HASELSTEINER, Horst, SUPPAN, Arnold, Innere Front. Militärassistenz, Widerstand und Umsturz in der Donaumonarchie 1918, 2 voll., Wien, Verlag für Geschichte und Politik, 1974; LEIDINGER, Hannes, MORITZ Verena, Gefangenschaft, Revolution, Heimkehr, cit., pp. 453-504. 
si affrontavano, sembrava impraticabile. Il piano proposto dalla Commissione per i prigionieri di Pietrogrado e adottato dal comando supremo del Baden, secondo cui il rimpatrio dei prigionieri lasciati indietro sarebbe dovuto avvenire con l'impiego di truppe austro-ungariche da inviare via treno ad est della Russia, venne accantonato per diverse ragioni88. L'intera impresa sembrava troppo rischiosa. Vi erano anche ufficiali che erano già stati prigionieri dei russi e che avrebbero dovuto, su incarico dei servizi segreti, valutare le possibilità di un rimpatrio di ulteriori soldati e ufficiali austroungarici. Le condizioni per questo genere di operazioni erano, come si può immaginare, molto pericolose e le relazioni di questi ufficiali non lasciavano molti dubbi in merito. Ufficiali austro-ungarici, la cui copertura non resse, vennero arrestati dall'Armata Rossa o dalle Guardie Rosse e, nei casi peggiori, eliminati. Lo stesse avvenne anche per mano di forze antibolsceviche ${ }^{89}$. La stragrande maggioranza dei prigionieri di guerra che si trovavano in Siberia poterono rientrare in patria, a dispetto delle misure messe in atto dai servizi segreti nel 1918, solo tra il 1920 e il $1921^{90}$. A quel punto la guerra di spie tra est e ovest aveva già assunto nuove forme.

\section{Conclusioni}

L’importanza dei prigionieri di guerra per i servizi segreti austro-ungarici durante la Prima guerra mondiale riflette da molteplici punti di vista le particolarità di quell'istituzione in un impero multietnico. Mentre nell'Austro-Ungheria, per esempio, la vicinanza tra i prigionieri russi e la propria popolazione slava minacciava la "sicurezza interna", i soldati sleali che si trovavano in prigionia in Russia impegnavano in modo particolare i servizi segreti. L" "età delle nazioni” arrivò anche in concomitanza con il ritorno dei prigionieri russi nell'ormai tramontato impero multinazionale zarista. Sulla base delle informazioni che i servizi segreti ottennero dalle loro attività in Russia venne deciso quali prigionieri russi rilasciare o meno sulla base dei rapporti di forza nelle loro aree di origine. Questo non è l'unico esempio che indica come gli sconvolgimenti in Russia influenzarono il lavoro dei servizi di informazione.

\footnotetext{
${ }^{88}$ MORITZ, Verena, Zwischen Nutzen und Bedrohung, cit., pp. 255-26o. Cfr. anche: Schreiben der 10. Kgf.-Abt. vom 11.7.1918, Zl. 131418, in ÖStA, KA, AOK Op. Abt. Heimkehrgruppe, Kt. 362.

${ }^{89}$ Cfr. RUTKOWSKI, Ernst, op. cit., pp. 15-78. A proposito dell'ipotesi di armare i prigionieri di guerra austro-ungarici in Russia e sui piani per la loro liberazione, si veda: LEIDINGER, Hannes, MORITZ, Verena, Gefangenschaft, Revolution, Heimkehr, cit., pp. 365-367.

${ }_{90}$ Sui rimpatri si veda: LEIDNGER, Hannes, Zwischen Kaiserreich und Rätemacht. Die deutschösterreichischen Heimkehrer aus russischer Kriegsgefangenschaft und die Organisation des österreichischen Kriegsgefangenen- und Heimkehrwesens 1917-1920, Wien, Diplomarbeit, 1995.
} 
La gran quantità di fonti negli archivi austriaci, assieme al significato che $\mathrm{i}$ prigionieri hanno per la ricerca sul lavoro di intelligence nella Prima guerra mondiale, si contrappone alla carenza di attenzione da parte della ricerca storica. Questo contributo ha cercato di mostrare come il lavoro dei servizi segreti si incrociò sotto molti punti di vista con le questioni relative ai prigionieri. Non sarebbe possibile, senza queste considerazioni, sviluppare un'auspicabile analisi complessiva dei servizi segreti dell' imperial-regio esercito nella Prima guerra mondiale; interesse nei confronti dei servizi segreti e dello spionaggio nella Grande guerra che, negli ultimi anni, è cresciuto in grande misura. 


\section{${ }^{*}$ L'autore}

Verena Moritz è docente presso le Università di Vienna e Salisburgo e coordinatrice del progetto "Prigionieri di guerra in Austria-Ungheria", finanziato dal consiglio nazionale delle ricerche austriaco. È anche membro della commissione storica austro-russa. Le sue principali aree di ricerca sono la Prima guerra mondiale nell'impero asburgico e in Russia, la storia della Russia e dell'Unione Sovietica, la storia dell'Austria tra il 1918 e il 1955, la storia delle relazioni internazionali, dello spionaggio nel XX secolo e della cinematografia.

URL: < http://www.studistorici.com/progett/autori/\#Moritz >

\section{Per citare questo articolo:}

MORITZ, Verena, «Prigionieri russi, prigionieri in Russia: detenzione, tradimento e spionaggio nella percezione e nelle strategie dei servizi segreti austroungarici (1914-1918)», Diacronie. Studi di Storia Contemporanea : La voce del silenzio: intelligence, spionaggio e conflitto nel XX secolo, 29/12/2016,

URL:<http://www.studistorici.com/2016/12/29/moritz_numero_28/ >

Diacronie Studi di Storia Contemporanea 0 www.diacronie.it

Risorsa digitale indipendente a carattere storiografico. Uscita trimestrale.

redazione.diacronie@hotmail.it

Comitato di redazione: Jacopo Bassi - Luca Bufarale - Antonio César Moreno Cantano - Deborah Paci - Fausto Pietrancosta - Alessandro Salvador - Matteo Tomasoni - Luca Zuccolo

Diritti: gli articoli di Diacronie. Studi di Storia Contemporanea sono pubblicati sotto licenza Creative Commons 3.0. Possono essere riprodotti e modificati a patto di indicare eventuali modifiche dei contenuti, di riconoscere la paternità dell'opera e di condividerla allo stesso modo. La citazione di estratti è comunque sempre autorizzata, nei limiti previsti dalla legge. 